\title{
Maintenance avelumab in metastatic urothelial cancer patients
}

\author{
Address for correspondence: \\ dr hab. n. med. Jakub Kucharz \\ Department of Genitourinary Oncology, \\ Maria Sklodowska-Curie National Research \\ Institute of Oncology \\ Roentgena 5 St., 02-781 Warsaw, Poland \\ e-mail: jakub.kucharz@pib-nio.pl
}

Translation: dr n. med. Dariusz Stencel Oncology in Clinical Practice

DOI: $10.5603 / \mathrm{OCP} .2021 .0021$

Copyright ( $\odot 2021$ Via Medica

ISSN 2450-1654

e-ISSN 2450-6478

\begin{abstract}
The treatment outcomes of patients with metastatic urothelial carcinoma remain poor. Despite the relatively high response rate to platinum-based chemotherapy, the median overall survival doesn't exceed 14 months. Immunotherapy with anti-PD-1/anti-PD-L1 antibodies in the second-line treatment shows significant activity but nearly $50 \%$ of patients are not eligible for such treatment because of poor performance status. Therefore, there is a need for new treatment strategies. In the phase III JAVELIN Bladder 100 clinical trial, the maintenance treatment with avelumab in patients who achieved disease control with platinum-based first-line chemotherapy resulted in prolongation of overall survival and progression-free survival with good safety profile.
\end{abstract} Key words: urothelial carcinoma, maintenance treatment, avelumab, immunotherapy

Oncol Clin Pract 2021; 17, 6: 291-294

\section{Introduction}

The prognosis of patients with metastatic urothelial carcinoma remains poor. The standard of first-line treatment is chemotherapy, preferable with cisplatin-based regimen due to the greatest therapeutic benefits [1] Despite the objective response rate (ORR) of $50 \%$ and disease control in approximately $80 \%$ of patients, the median progression-free survival (PFS) is approximately 9 months, and the median overall survival (OS) is approximately 14 months [2]. Long-term disease control is achieved in approximately $10-15 \%$ of patients with metastases confined to lymph nodes [2]. In patients with contraindications to cisplatin, carboplatin-based regimens are used, but this treatment is associated with worse outcomes [3]. In patients who do not qualify to chemotherapy and have an expression of programmed death ligand-1 (PD-L1), it is also possible to use immune checkpoint inhibitors, such as atezolizumab (PD-L1 $\geq 5 \%$ ) or pembrolizumab [in patients with a combined positive score $(\mathrm{CPS}) \geq 10][4,5]$. Immuno- therapy has undoubtedly a well-established role in the second-line treatment after failure of platinum-based chemotherapy [2]. The European Medicines Agency (EMA) has registered pembrolizumab, atezolizumab and nivolumab in this indication [6-8].

Other second-line treatment strategies include rechallenge with platinum-based chemotherapy (if the first-line response was achieved and time to re-treatment is longer than 12 months), erdafitinib (in case of confirmed FGFR2 or FGFR3 gene rearrangement), or enfortumab vedotin (antibody-drug conjugate directed against nectin-4) [2].

It should be emphasized that only about $50-60 \%$ of patients who receive systemic treatment for metastatic urothelial carcinoma are eligible for second-line treatment, which is usually a consequence of the high dynamics of the disease and a significant deterioration of the general condition [9].

Therefore, it is necessary to search for new therapeutic strategies that can improve the prognosis of patients with metastatic urothelial carcinoma.

\section{Received: 21.05.2021 Accepted: 21.05.2021 Early publication date: 04.11.2021}

This article is available in open access under Creative Common Attribution-Non-Commercial-No Derivatives 4.0 International (CC BY-NC-ND 4.0) license, allowing to download articles and share them with others as long as they credit the authors and the publisher, but without permission to change them in any way or use them commercially. 


\section{Maintenance treatment}

The concept of maintenance treatment is based on the continuation of therapy at a lower intensity after the disease control is achieved by earlier treatment [10]. It is aimed at delaying disease progression, worsening of clinical status, and prolonging OS. The drugs used in maintenance therapy should have good tolerability and a favourable safety profile. There are two strategies for maintenance therapy. The first strategy - continuation maintenance - is to continue the administration of one of the drugs used during induction therapy (an example is fluoropyrimidine monotherapy in patients with metastatic colorectal carcinoma who have responded to induction therapy with a multi-drug regimen) [11]. The second strategy — switch maintenance — is based on monotherapy with a drug not used in the current regimen [an example is the use of poly(ADP-ribose) polymerase (PARP) inhibitors] in patients with a serous ovarian, fallopian tube or peritoneal cancer with response to platinum-based chemotherapy [12-15].

\section{Maintenance treatment in patients with metastatic urothelial carcinoma}

Attempts have been made in the past to use maintenance treatment strategies in patients with metastatic urothelial carcinoma. The value of such approach was assessed in the phase II MAJA study (SOGUG 2011/02) [16]. A group of 88 patients who achieved disease control with platinum-gemcitabine chemotherapy with platinum and gemcitabine (4-6 cycles) were randomized to receive either vinflunine monotherapy (45 subjects) or best supportive care (BSC) (43 subjects). There was an increase in the median PFS [6.2 versus 4 months; hazard ratio $(\mathrm{HR})=0.59,95 \%$ confidence interval (CI): 0.37-0.96]. However, this management was not approved due to the significant toxicity of vinflunine.

In another study, the efficacy of lapatinib $(n=116)$ was assessed versus placebo $(\mathrm{n}=116)$ in patients with overexpression of human epidermal growth factor receptors-1-2 (HER-1-2), with disease control after 4-8 cycles of platinum-based chemotherapy [17]. There was no benefit from lapatinib therapy (median PFS 4.5 versus 5.1 months; $\mathrm{HR}=1.07,95 \% \mathrm{CI}: 0.81-1.43$; OS 12.6 versus 12 months; $\mathrm{HR}=0.96,95 \% \mathrm{CI}: 0.70-1.31$ ). Also, sunitinib was not active in maintenance therapy [18]. Median PFS was 2.9 months in the active treatment group (95\% CI: $2.4-6.3$ ) versus 2.7 months in the placebo group (95\% CI: $2.5-7.2)(\mathrm{HR}=1,0.95 \% \mathrm{CI}: 0.6-1.8)$.

On the other hand, the HCRN GU14-182 study assessed the efficacy of pembrolizumab in patients who achieved at least disease stabilization after platinum-based chemotherapy (1-8 cycles) [19]. Patients were randomized in a $1: 1$ ratio to pembrolizumab maintenance treatment or placebo. The median PFS was 5.4 months in the pembrolizumab arm versus 3.2 months in the placebo arm $(\mathrm{HR}=0.64, \mathrm{p}=0.038)$. The study design allowed patients randomized to placebo to cross over to the active treatment arm after disease progression and finally, $52 \%$ recieved pembrolizumab.

\section{Avelumab treatment for metastatic urothelial carcinoma}

Avelumab is a human monoclonal IgG1 antibody directed against PD-L1 [20]. A feature that distinguishes avelumab from other anti-PD-1/PD-L1 antibodies is its potential to induce antibody-dependent cellular cytotoxicity (ADCC), which has been confirmed in preclinical studies [21], however, there is no data available indicating the clinical significance of this difference. Avelumab activity in the treatment of patients with locally advanced or metastatic urothelial carcinoma was confirmed in a cohort of patients with this diagnosis included in the phase I JAVELIN Solid Tumour study [22]. Treatment was associated with a good safety profile, the objective response rate with a follow-up period of at least 6 months was $17 \%$ (95\% CI: 11-24), the median duration of response was not reached [22]. On this basis, the US Food and Drug Administration (FDA) approved avelumab, using the Accelerated Approval Pathway, for the treatment of patients with locally advanced or metastatic urothelial cancer who have progressed during or after platinum-based chemotherapy or within 12 months of completion neoadjuvant or adjuvant treatment.

The effectiveness of avelumab in maintenance treatment was assessed in the phase III JAVELIN Bladder 100 study [23]. The study included 700 patients who were randomized in a 1:1 ratio to treatment with avelumab or BSC. Treatment was initiated 4-10 weeks after chemotherapy completion. Patients received avelumab at a dose of $10 \mathrm{mg} / \mathrm{kg}$ every 14 days (the first 4 cycles with premedication with antihistamine and paracetamol). Treatment was continued until disease progression, unacceptable toxicity, or consent withdrawal. The primary endpoints were OS in the general population and in patients with PD-L1 expression. Secondary endpoints were PFS, ORR, time to response, duration of response, disease control rate, and safety. The median OS in the general population was 21.4 months (95\% CI: 18.9-26.1) in patients treated with avelumab versus 14.3 months (95\% CI: $12.9-17.9)$ in the BSC group (HR 0.69, 95\% CI: 0.56-0.86, $\mathrm{p}<0.01$ ). In patients with PD-L1 expression, the median OS was not reached in the avelumab group (20.3 - NR) and was 17.1 months (13.5-23.7) in the BSC group (HR 0,56; 95\% CI, $0,40-0-79)$. The median PFS was in the general population in patients treated with avelumab 3.7 months (95\% CI: 3.5-5.5) versus 2.0 months in the BSC group 
(95\% CI: 1, 9-2.7) (HR 0.62; 95\% CI, 0.52-0.75), while in the group with PD-L1 expression - 5.7 months (95\% CI, 3.7-7.4) and 2.1 months (95\% CI, 1.9-3.5) (HR 0,56; 95\% CI, 0.43-0.73), respectively. Next-line treatment was administered to $42.3 \%$ of patients in the group treated with avelumab and $61.7 \%$ of patients in the BSC group (43.7\% received anti-PD1/anti-PD-L1 antibody). Immune-related adverse events (irAEs) were reported in $29.4 \%$ of patients receiving avelumab; in $7 \%$ of patients there were CTCAE (Common Terminology Criteria for Adverse Events) grade 3 events, but no grade 4 complications were found. Thyroid dysfunction was the most common irAE. The use of glucocorticosteroids at a dose of $\geq 40 \mathrm{mg}$ of prednisone (or equivalent) was required in $9 \%$ of patients treated with avelumab.

In a subgroup analysis, the benefit of treatment with avelumab was found in all patients, regardless of PDL1 expression, presence of visceral metastases, chemotherapy regimen (gemcitabine with cisplatin, gemcitabine with carboplatin) and the response obtained (stabilization, partial response, complete response).

During the 2021 Genitourinary Cancers Symposium (ASCO GU), an analysis of data from the JAVELIN Bladder 100 study was also presented, which assessed the benefit of avelumab treatment depending on the duration of first-line chemotherapy and the number of cycles administered (4-6). The benefit of maintenance treatment was found in all groups of patients [24].

US FDA and EMA approved avelumab for maintenance treatment of patients who have not progressed after platinum-based first-line chemotherapy.

\section{Summary}

The results of the JAVELIN Bladder 100 study are extremely important in the context of optimizing the strategy and sequence of treatment in patients with metastatic urothelial carcinoma. As mentioned, approximately $50 \%$ of patients who fail first-line treatment will not be eligible for further treatment. In this context, early use of maintenance immunotherapy after disease control by chemotherapy is warranted. From a biological point of view, the benefit of such a strategy may be related to the immunomodulatory effects of chemotherapy including depletion of $\mathrm{T}$ regulatory lymphocytes and myeloid-derived suppressor cells, as well as increased NK lymphocyte activity, neoantigens release and PDL1 expression on tumour cells [25-29]. However, it should be taken into account that in approximately $10-15 \%$ of patients, long-term disease control can be achieved with chemotherapy alone [2], and in this group maintenance treatment will not be associated with any additional benefit. At present, however, no factors are allowing for the identification of these patients.
As part of the search for the optimal procedure, attempts have been made to combine chemotherapy with immunotherapy in first-line treatment. However, the results of KEYNOTE-361 [30] and IMvigor-130 [31] studies presented so far do not justify changing clinical practice (IMvigor-130 study showed benefit only for PFS with immature data for OS). The negative outcomes of clinical trials with chemoimmunotherapy in metastatic urothelial carcinoma may result - between others - from the fact that approximately $20 \%$ of patients in this population experience primary chemoresistance, that is associated with particularly poor prognosis and reduced benefit of immunotherapy.

Improving the treatment outcomes in patients with metastatic urothelial carcinoma is possible thanks to the introduction of new, active therapeutic strategies into clinical practice. The natural need is to determine the optimal treatment sequence and the possibility of combining them, e.g., with chemotherapy. The use of avelumab in the maintenance treatment of patients with metastatic urothelial carcinoma is a valuable therapeutic strategy, and the results of the JAVELIN Bladder 100 study provide the basis for defining a new standard of care in this group of patients, which was reflected in the recommendations of the European Society of Clinical Oncology (ESMO) [32], European Association of Urology (EAU) [33], and National Comprehensive Cancer Network (NCCN) [34].

\section{Conflict of interest}

Advisory and lecture fees from Merck and Pfizer. Travel grants from Pfizer.

\section{References}

1. Bellmunt J, Orsola A, Leow JJ, et al. ESMO Guidelines Working Group. Bladder cancer: ESMO Practice Guidelines for diagnosis, treatment and follow-up. Ann Oncol. 2014; 25 Suppl 3: iii40-iii48, doi: 10.1093/annonc/mdu223, indexed in Pubmed: 25096609

2. von der Maase H, Hansen SW, Roberts JT, et al. Gemcitabine and cisplatin versus methotrexate, vinblastine, doxorubicin, and cisplatin in advanced or metastatic bladder cancer: results of a large, randomized, multinational, multicenter, phase III study. J Clin Oncol. 2000; 18(17): 3068-3077, doi: 10.1200/JCO.2000.18.17.3068, indexed in Pubmed: 11001674

3. Santis MDe, Bellmunt J, Mead G, et al. Randomized Phase II/II Trial Assessing Gemcitabine/Carboplatin and Methotrexate/Carboplatin/Vinblastine in Patients With Advanced Urothelial Cancer Who Are Unfit for Cisplatin-Based Chemotherapy: EORTC Study 30986. J Clin Oncol. 2012; 30(2): 191-199, doi: 10.1200/jco.2011.37.3571.

4. Balar A, Galsky M, Rosenberg J, et al. Atezolizumab as first-line treatment in cisplatin-ineligible patients with locally advanced and metastatic urothelial carcinoma: a single-arm, multicentre, phase 2 trial. The Lancet. 2017; 389(10064): 67-76, doi: 10.1016/s01406736(16)32455-2.

5. Balar AV, Castellano D, O'Donnell PH, et al. First-line pembrolizumab in cisplatin-ineligible patients with locally advanced and unresectable or metastatic urothelial cancer (KEYNOTE-052): a multicentre, single-arm, phase 2 study. Lancet Oncol. 2017; 18(11): 1483-1492, doi: 10.1016/S1470-2045(17)30616-2, indexed in Pubmed: 28967485. 
6. Bellmunt J, de Wit R, Vaughn DJ, et al. KEYNOTE-045 Investigators. Pembrolizumab as Second-Line Therapy for Advanced Urothelial Carcinoma. N Engl J Med. 2017; 376(11): 1015-1026, doi 10.1056/NEJMoa1613683, indexed in Pubmed: 28212060.

7. Powles T, Durán I, van der Heijden MS, et al. Atezolizumab versus chemotherapy in patients with platinum-treated locally advanced or metastatic urothelial carcinoma (IMvigor211): a multicentre, open-label, phase 3 randomised controlled trial. Lancet. 2018; 391(10122) 748-757, doi: 10.1016/S0140-6736(17)33297-X, indexed in Pubmed: 29268948.

8. Sharma P, Retz M, Siefker-Radtke A, et al. Nivolumab in metastatic urothelial carcinoma after platinum therapy (CheckMate 275): a multicentre, single-arm, phase 2 trial. Lancet Oncol. 2017; 18(3): 312-322, doi: 10.1016/S1470-2045(17)30065-7, indexed in Pubmed: 28131785.

9. Ma'koseh M, Salam M, Al-Wardat R, et al. Metastatic urothelial tumors progressing following first line chemotherapy: prognostic factors and importance of second line chemotherapy. J Clin Oncol. 2016; 34(15 suppl): e16036-e16036, doi: 10.1200/jco.2016.34.15_suppl.e16036.

10. Grivas P, Monk BJ, Petrylak D, et al. Immune Checkpoint Inhibitors as Switch or Continuation Maintenance Therapy in Solid Tumors: Rationale and Current State. Target Oncol. 2019; 14(5): 505-525, doi: 10.1007/s11523-019-00665-1, indexed in Pubmed: 31535338

11. Sonbol MB, Mountjoy LJ, Firwana B, et al. The Role of Maintenance Strategies in Metastatic Colorectal Cancer: A Systematic Review and Network Meta-analysis of Randomized Clinical Trials. JAMA Oncol. 2020; 6(3): e194489, doi: 10.1001/jamaoncol.2019.4489, indexed in Pubmed: 31855256

12. Pujade-Lauraine E, Ledermann JA, Selle F, et al. Olaparib tablets as maintenance therapy in patients with platinum-sensitive, relapsed ovarian cancer and a BRCA1/2 mutation (SOLO2/ ENGOT-Ov21): a double-blind, randomised, placebo-controlled, phase 3 trial. Lancet Oncol. 2017; 18: 1274-1284.

13. Mirza MR, Monk BJ, Herrstedt J, et al. ENGOT-OV16/NOVA Investigators. Niraparib Maintenance Therapy in Platinum-Sensitive, Recurrent Ovarian Cancer. N Engl J Med. 2016; 375(22): 2154-2164, doi: 10.1056/NEJMoa1611310, indexed in Pubmed: 27717299.

14. Friedlander M, Moore KN, Colombo N, et al. Maintenance Olaparib in Patients with Newly Diagnosed Advanced Ovarian Cancer. N Eng J Med. 2018; 379(26): 2495-2505, doi: 10.1056/NEJMoa1810858, indexed in Pubmed: 30345884

15. Coleman RL, Oza AM, Lorusso D, et al. Rucaparib mainte- nance treatment for recurrent ovarian carcinoma after response to platinum therapy (ARIEL3): a randomised, double-blind, placebo-controlled, phase 3 trial. Lancet. 2017; 390: 1949-1961.

16. García-Donas J, Font A, Pérez-Valderrama B, et al. Maintenance therapy with vinflunine plus best supportive care versus best supportive care alone in patients with advanced urothelial carcinoma with a response after first-line chemotherapy (MAJA; SOGUG 2011/02): a multicentre randomised, controlled, open-label, phase 2 trial. Lancet Oncol. 2017 18(5), doi: 10.1016/s1470-2045(17)30242-5

17. Powles T, Huddart RA, Elliott T, et al. Phase III, Double-Blind, Randomized Trial That Compared Maintenance Lapatinib Versus Placebo After First-Line Chemotherapy in Patients With Human Epidermal Growth Factor Receptor 1/2-Positive Metastatic Bladder Cancer. J Clin Oncol. 2017; 35(1): 48-55, doi: 10.1200/JCO.2015.66.3468, indexed in Pubmed: 28034079.

18. Grivas PD, Daignault S, Tagawa ST, et al. Double-blind, randomized phase 2 trial of maintenance sunitinib versus placebo after response to chemotherapy in patients with advanced urothelial carcinoma. Cancer. 2014; 120(5): 692-701, doi: 10.1002/cncr.28477, indexed in Pubmed: 24249435.

19. Galsky MD, Mortazavi A, Milowsky MI, et al. Randomized Double-Blind Phase II Study of Maintenance Pembrolizumab Versus Placebo After First-Line Chemotherapy in Patients With Metastatic Urothelial Cancer.
J Clin Oncol. 2020; 38(16): 1797-1806, doi: 10.1200/JCO.19.03091, indexed in Pubmed: 32271672

20. Boyerinas B, Jochems C, Fantini M, et al. Antibody-Dependent Cellular Cytotoxicity Activity of a Novel Anti-PD-L1 Antibody Avelumab (MSB0010718C) on Human Tumor Cells. Cancer Immunol Res. 2015; 3(10): 1148-1157, doi: 10.1158/2326-6066.CIR-15-0059, indexed in Pubmed: 26014098

21. Juliá EP, Amante A, Pampena MB, et al. Avelumab, an lgG1 anti-PD-L1 Immune Checkpoint Inhibitor, Triggers NK Cell-Mediated Cytotoxicity and Cytokine Production Against Triple Negative Breast Cancer Cells. Front Immunol. 2018; 9: 2140, doi: 10.3389/fimmu.2018.02140, indexed in Pubmed: 30294328.

22. Patel MR, Ellerton J, Infante JR, et al. Avelumab in metastatic urothelial carcinoma after platinum failure (JAVELIN Solid Tumor): pooled results from two expansion cohorts of an open-label, phase 1 trial. Lancet Oncol. 2018; 19(1): 51-64, doi: 10.1016/S1470-2045(17)30900-2, indexed in Pubmed: 29217288.

23. Powles T, Park SeH, Voog E, et al. Avelumab Maintenance Therapy for Advanced or Metastatic Urothelial Carcinoma. N Engl J Med. 2020; 383(13): 1218-1230, doi: 10.1056/NEJMoa2002788, indexed in Pubmed: 32945632

24. Loriot $Y$, Powles T, Durán MC, et al. Avelumab (Ave) first-line (1L) maintenance plus best supportive care (BSC) versus BSC alone for advanced urothelial carcinoma (UC): JAVELIN Bladder 100 subgroup analysis based on duration and cycles of $1 \mathrm{~L}$ chemotherapy. J Clin Oncol. 2021; 39(6_suppl): 438-438, doi: 10.1200/jco.2021.39.6_ suppl.438.

25. Hato S, Khong A, Vries Ide, et al. Molecular Pathways: The Immunogenic Effects of Platinum-Based Chemotherapeutics. Clin Cancer Res. 2014; 20(11): 2831-2837, doi: 10.1158/1078-0432. ccr-13-3141.

26. Kyi C, Postow MA. Immune checkpoint inhibitor combinations in solid tumors: opportunities and challenges. Immunotherapy. 2016; 8(7): 821-837, doi: 10.2217/imt-2016-0002, indexed in Pubmed: 27349981.

27. Luo M, Fu L. The effect of chemotherapy on programmed cell death 1/programmed cell death 1 ligand axis: some chemotherapeutical drugs may finally work through immune response. Oncotarget. 2016; 7(20): 29794-29803, doi: 10.18632/oncotarget.7631, indexed in Pubmed: 26919108

28. Song Z, Yu X, Zhang Y. Altered expression of programmed death-ligand 1 after neo-adjuvant chemotherapy in patients with lung squamous cell carcinoma. Lung Cancer. 2016; 99: 166-171, doi: 10.1016/j. lungcan.2016.07.013, indexed in Pubmed: 27565935.

29. Zitvogel L, Apetoh L, Ghiringhelli F, et al. The anticancer immune response: indispensable for therapeutic success? J Clin Invest. 2008; 118(6): 1991-2001, doi: 10.1172/JCl35180, indexed in Pubmed: 18523649

30. Alva A, Csoszi T, Ozguroglu M, et al. Pembrolizumab combined with chemotherapy vs chemotherapy alone as first-line therapy for advanced urothelial carcinoma: KEYNOTE-361. Pre- sented at the European Society for Medical Oncology (ESMO) Virtual Congress September 18-21, 2020

31. Galsky MD, Arija JÁ, Bamias A, et al. IMvigor130 Study Group. Atezolizumab with or without chemotherapy in metastatic urothelial cancer (IMvigor130): a multicentre, randomised, placebo-controlled phase 3 trial. Lancet. 2020; 395(10236): 1547-1557, doi: 10.1016/S01406736(20)30230-0, indexed in Pubmed: 32416780.

32. eUpdate - Bladder Cancer Treatment Recommendations Published: 16 July 2020. https://www.esmo.org/guidelines/genitourinary-cancers/bladder-cancer.

33. https://uroweb.org/guideline/bladder-cancer-muscle-invasive-and-metastatic.

34. NCCN Bladder Cancer Guideliness - Version 3.2021. www.nccn.org. 